\title{
Progress in home-based treatment
}

\author{
P. McGarry* \\ Belfast Home Treatment Team, Belfast, Northern Ireland
}

\begin{abstract}
Home-based treatment has been promoted as an alternative to hospital admission over the last 30 years, with the system in England being particularly well developed. There is less stigma, patients and families appear to prefer treatment at home, and there has been a significant reduction in the number of acute beds. In more recent years, there have been criticisms of the lack of effect on reducing hospital admissions and concerns have also been raised about patient safety. Home-based treatment is being further developed on the island of Ireland, and this will hopefully be influenced by current and future local research.
\end{abstract}

Key words: Centre for suicide prevention, crisis response home treatment, home treatment accreditation service, risk assessment.

\section{Introduction}

The term 'Social psychiatry' became current in Holland in the 1920s. In Leiden, Jelgersma (Oosterhuis, 2004) took the then radical view that psychosis did not in itself constitute a sufficient cause for hospitalisation. In Amsterdam in the 1930s, Querido (1935) developed a comprehensive social psychiatry approach, which involved teams of doctors and nurses offering crisis intervention and domiciliary visits, with every effort being made to provide alternatives to hospital admission.

\section{Australian Pioneer of Home Treatment}

The modern iteration of Home Treatment was led by the Australian Psychiatrist John Hoult who developed his model in Sydney. Hoult noted that the outcomes for patients were better than the traditional approach, the costs were less and - crucially - patients and families were both found to prefer Home Treatment (Hoult et al., 1984).

In 1995 Hoult came to the UK, where Home Treatment had been pioneered in the Birmingham district of Sparkbrook (one of the most deprived area of the UK) by Dean and Gadd. Previously their research (Dean \& Gadd, 1990) confirmed - contrary to the expectations of some colleagues - that Home Treatment was preferred by relatives as well as patients.

\section{Development in the UK}

During the 1990s, services were set up in a number of areas including Bradford and Barnet, but there was a real step change in 2000 when the Department of Health mandated that every local health district in

\footnotetext{
* Address for correspondence: Dr. P. McGarry, Consultant Psychiatrist, Belfast Home Treatment Team, Fairview 2, Crumlin Road, Belfast, Northern Ireland.

(Email: philipj.mcgarry@belfasttrust.hscni.net)
}

England and Wales had to develop a Home Treatmentbased service (called Crisis Resolution Home Treatment or CRHT) (Department of Health (DOH), 2000). It is of note that the document was very specific in stating that the role of Home Treatment was to prevent the admission to hospital of patients suffering from severe mental illnesses such as schizophrenia, bipolar disorder and severe depression. It was not primarily for people with anxiety disorders, substance misuse, those who had self-harmed in the absence of severe mental illness or in crisis due to relationship problems. CRHT was expected to 'gatekeep' access to acute beds, and be available around the clock. This commitment to treating severe illness was reiterated in a 2006 report by the Care Services Improvement Partnership, CSIP (Onyett et al., 2006) which stated that there needed to be a separate liaison service to Emergency Departments (EDs), on the basis that the large majority of patients who present to ED's do not have severe mental illness nor require admission to an acute ward, with an estimate of perhaps $10 \%$ requiring Home Treatment. The authors stated: 'CRHT ought not to become a de facto liaison service'.

In 2007, the National Audit Office noted that the number of admissions had declined by $21 \%$ since 2000 , with a larger fall in areas with a $24 / 7$ CRHT service (Morgan, 2007). The report reaffirmed that Home Treatment Services were primarily designed for the treatment of people who were severely mentally ill, in order to prevent admission to hospital, and to facilitate early discharge (the latter being an effective way of reducing length of stay).

These three key documents all emphasised the key components of a 'high fidelity' service necessary to achieve the essential reductions in bed usage (CRHT could only be funded by closing in-patient beds). These were 'gatekeeping' all admissions, providing a $24 / 7$ service, facilitating early discharge and having 
significant medical input. 'Gatekeeping' requires CRHT to have the responsibility for assessing and accepting all admissions to hospital, including - ideally - detentions.

Middleton et al. (2008) carried out a survey of Home Treatment Teams across England and noted a large variation in the availability of medical staff, particularly consultants. A few teams had no medical input at all. Inevitably this affected the ability of teams to manage and crucially not admit to hospital - ill patients. Full gatekeeping and the provision of $24 / 7$ services has never been achieved in many CRHT teams. Johnston (2013) reported that only $39 \%$ of teams have a $24 / 7$ service and only $30 \%$ assess all admissions to hospital. She emphasised that despite the different practices across England, there was general agreement that the focus should remain on people 'at risk of hospital admission'.

England's experience has shown how difficult it is, even with strong direction from the centre, for a major change in service provision to be implemented across a whole country.

In recent years the Royal College of Psychiatrists has set up the Home Treatment Accreditation Scheme (HTAS), designed to drive up standards and encourage a greater degree of consistency in service provision.

\section{Criticisms of Home Treatment}

Critics of Home Treatment have expressed concerns about a fragmentation in care, with patients moving between a number of consultants during acute episodes of illness. The Centre for Suicide Prevention in Manchester has documented (Hunt et al., 2016) that while there was a major reduction in the number of in-patient suicides from 2003, there was a significant increase in the number of suicides in Home Treatment. However, in the context of a much larger number of patients being treated by CRHT, there was actually a reduction of $18 \%$ in the suicide rate between 2003 and 2011. This issue is currently a major focus of research by the Manchester team.

Over recent years, the evidence for the costeffectiveness of Home Treatment has been somewhat more mixed than in earlier studies. Jethwa et al. (2007) reported a $37.5 \%$ reduction in admissions, while Johnson (2013) found fewer admissions post crisis. On the other hand, Jacobs and Barrenho (2011) found no impact of CRHT on admissions. Murphy et al. (2015) concluded for the Cochrane collaboration that while the research evidence was very limited: 'Home Treatment appears to be a viable and acceptable way of treating people with serious mental illness'. The review also noted that Home Treatment was more acceptable to patients and 'is less burdensome to relatives', while there was evidence that it can reduce the number of repeat admissions and lead to improvement in patients' mental health.

In Belfast, Home Treatment was set up in 2006. It has always worked on a $24 / 7$ basis. In the year after, it took over responsibility for gatekeeping all admissions in 2009 , the number of admissions dropped by $27 \%$. Belfast has achieved a reduction of over $40 \%$ in acute psychiatric beds since 2009. The team has four consultants, including an old age psychiatrist. Belfast also has a self-harm/personality disorder team, and an unscheduled care team which assesses patients presenting to the EDs and many of the crisis patients referred by general practitioner's (GP), thus allowing Home Treatment to focus on the most severely ill patients.

Forbes et al. (2010) noted that the number of admissions in Midlothian had not fallen, despite the development of Home Treatment. However, this team only had a part-time staff grade psychiatrist. The author also explicitly noted the heightened concern of staff about risk, following the introduction of risk assessment tools for every patient, which may have lowered the threshold for admission. The NICE Guidelines (2011) indicate that risk assessment forms do not have any utility in predicting risk for individual patients, yet despite this, they appear to be mandated by most services. The current 'culture of risk' may be compromising the efforts of the last 75 years to try and care for patients in the least restrictive setting, and if so this is much to be regretted.

\section{Ireland}

In Ireland, some of the pioneers of Home Treatment were Dr Ian Daly in Dublin and Dr John Owens in Cavan Monaghan in the 1990s. The success of Cavan Monaghan was such that the Department of Health in Northern Ireland came to Dr Owens for advice when setting up its own services.

In Northern Ireland, the first Crisis Response Team was set up in the Homefirst Trust in 2005. With the reorganisation to five Trusts in April 2007, each Trust was mandated to provide Home Treatment. As has been the case elsewhere, different models of care have been set up across the five Trusts, although they do appear to be broadly similar.

Morrow et al. (2016) reported on a novel acute crisis service in Derry, which integrated in-patient beds, acute day care and Home Treatment. The introduction of the new service resulted in fewer admissions, a reduction in bed occupancy and a shorter length of stay.

In the current issue, there are two papers from the Republic of Ireland. Lavelic reports on a new service in North Cork. It does not yet gatekeep the beds, nor 
provide $24 / 7$ availability, but it has managed a very significant number of patients. Lavelic notes that the proportion of patients with psychosis or mania $(10 \%)$ is much lower than the 37\% reported in the Cavan service (Nwachukwu et al., 2013), while 56\% of referrals were from GPs and $16 \%$ from mental health teams.

O'Keefe and Russell describe the results of an allIreland survey of Home Treatment Services. While the response was not complete, the large majority of services did respond and a lot of very useful information has been gathered. The survey reflected the work in England and Wales that staffing levels and representations of different disciplines varied widely. While the survey was not able to look at outcome measures or provide details regarding bed usage, it is noteworthy that the significant majority of clinical directors were of the view that Home Treatment is cost-effective, and also that both patients and carers are perceived as preferring the option of treatment at home to admission to a psychiatric ward.

The paper concludes with a proposal to set up an allIreland forum on Home-Based Treatment. This is likely to find favour not only with psychiatrists but with all the other professionals and managers in-home treatment teams in Ireland. O'Keefe and Russell deserve full support in this enterprise. Hopefully, one outcome of the papers in this edition will be further research and service development in Home Based Treatment across Ireland.

\section{Conflicts of Interest}

Philip McGarry is Consultant Psychiatrist and Lead Clinician with the Belfast Home Treatment Team.

\section{Ethical standards}

The author asserts that all procedures contributing to this work comply with the ethical standards of the relevant national and institutional committee on human experimentation with the Helsinki Declaration of 1975 , as revised in 2008.

\section{Financial support}

This article received no specific grant from any funding agency, commercial or not-for-profit sectors.

\section{References}

Dean C, Gadd E (1990). Home treatment for acute psychiatric illness. BMJ 301 (6759): 1021-1023.
Department of Health (2000). The NHS Plan: A Plan for Investment, A Plan for Reform (https:/ /navigator.health. org.uk/content/nhs-plan-plan-investment-plan-reform2000). Accessed 17 January 2018.

Forbes N, Cash H, Lawrie S (2010). Intensive home treatment, admission rates and use of mental health legislation. The Psychiatrist 34, 522-524.

Hoult J, Rosen A, Reynolds I (1984). Community orientated treatment compared to psychiatric hospital orientated treatment. Social Science E Medicine 18, 1005-1010.

Hunt I, Appleby L, Kapur N (2016). Suicide under crisis resolution home treatment - a key setting for patient safety. BJPsych Bulletin 40, 172-174.

Jacobs R, Barrenho E (2011). Impact of crisis resolution and home treatment teams on psychiatric admissions in England. British Journal of Psychiatry 199, 71-76.

Jethwa K, Galappathie N, Hewson P (2007). Effects of a crisis resolution and home treatment team on in-patient admissions. Psychiatric Bulletin 31, 170-172.

Johnson S (2013). Crisis resolution and home treatment teams: an evolving model. Advances in Psychiatric Treatment 19, 115-123.

Middleton H, Glover G, Onyett S, Linde K (2008). Crisis resolution/home treatment teams, gate-keeping and the role of the consultant psychiatrist. Psychiatric Bulletin 32, 378-379.

Morgan S. Are crisis resolution and home treatment services seeing the patients they are supposed to see? National Audit Office, 2007.

Morrow R, McGlennon D, McDonnell C (2016). A novel mental health crisis service - outcomes of inpatient data. Ulster Med J 85, 13-17.

Murphy S, Irving C, Adams C, Waqar M (2015). Crisis intervention for people with severe mental illnesses. Cochrane Database of Systematic Reviews. (http:/ / www.cochrane. org/CD001087/SCHIZ_crisis-intervention-people-severemental-illness). Accessed 17 January 2018.

National Institute for Healthcare and Excellence (2011). Selfharm in over 8s-long-term management (CG133) (https:/ / www.nice.org.uk/guidance/cg133). Accessed 17 January 2018.

Nwachukwu I, Nkire N, Russell V (2013). Profile and activities of a rural home-based psychiatric treatment service in Ireland. International Journal of Psychiatry in Clinical Practice 18, 125-130.

Onyett S, Linde K, Glover G, Floyd S, Bradley S, Middleton H (2006). A National Survey of Crisis Resolution Teams in England. Care Services Improvement Partnership, Department of Health \& University of the West of England. (http:/ / eprints.nottingham.ac.uk/789/1/national-crisisresolution-team-survey.pdf). Accessed 17 January 2018.

Oosterhuis H (2004). Between institutional psychiatry and mental health care: social psychiatry in The Netherlands, 1916-2000. Medical History 48, 413-428.

Querido A (1935). Community mental hygiene in the city of Amsterdam. Mental Hygiene 19, 177-195. 